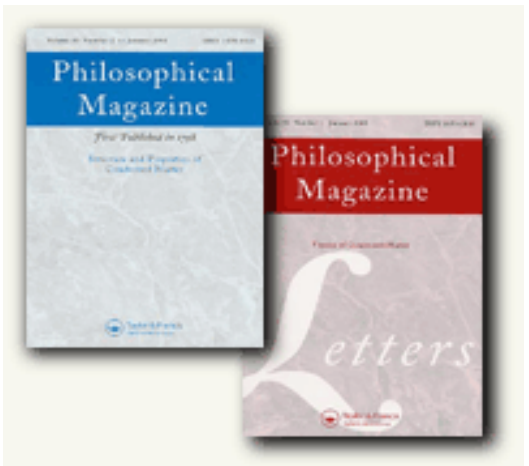

\title{
Friction between incommensurate crystals
}

\begin{tabular}{|c|c|}
\hline Journal: & Philosophical Magazine \& Philosophical Magazine Letters \\
\hline Manuscript ID: & TPHM-06-Feb-0042.R1 \\
\hline Journal Selection: & Philosophical Magazine \\
\hline $\begin{array}{r}\text { Date Submitted by the } \\
\text { Author: }\end{array}$ & 19-Jun-2006 \\
\hline Complete List of Authors: & $\begin{array}{l}\text { Friedel, Jacques; Université Paris-Sud, Orsay, Laboratoire de } \\
\text { Physique des Solides } \\
\text { Gennes, Pierre-Gilles; College de France; Institut Curie, Recherche }\end{array}$ \\
\hline Keywords: & graphite, incommensurate structures, tribology \\
\hline Keywords (user supplied): & Grain boundaries \\
\hline \multicolumn{2}{|c|}{$\begin{array}{l}\text { Note: The following files were submitted by the author for peer review, but cannot be converted } \\
\text { to PDF. You must view these files (e.g. movies) online. }\end{array}$} \\
\hline $\begin{array}{l}\text { Gennes MS-TPHM-06-Feb-00 } \\
\text { Gennes Figure captions } 26.6 \text {. }\end{array}$ & $\begin{array}{l}\text { 42RevisedVersion.tex } \\
\text { 06.tex }\end{array}$ \\
\hline
\end{tabular}

\section{s scholaroNE" Manuscript Central}




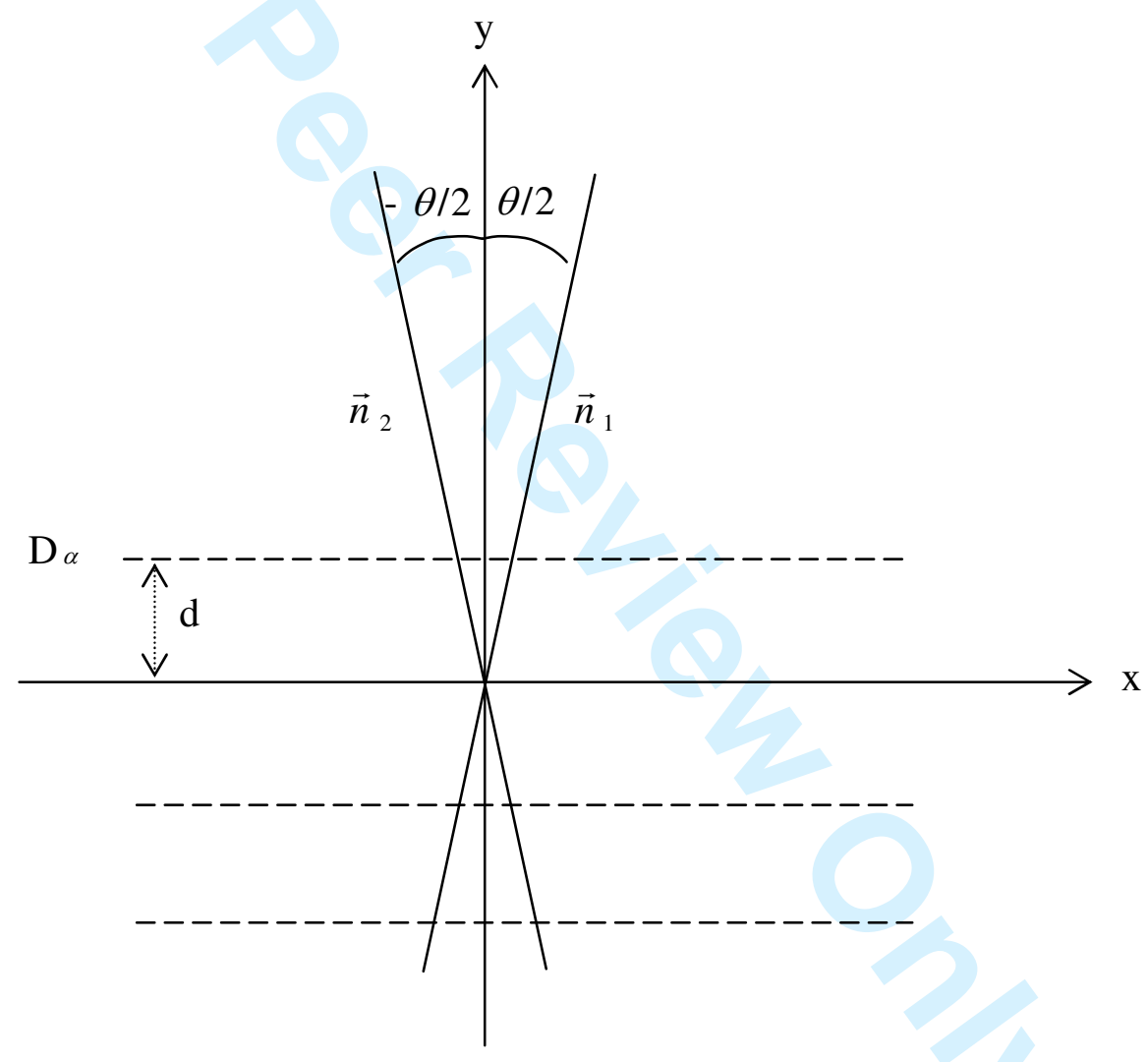

Fig. 1 
Fig. 2 

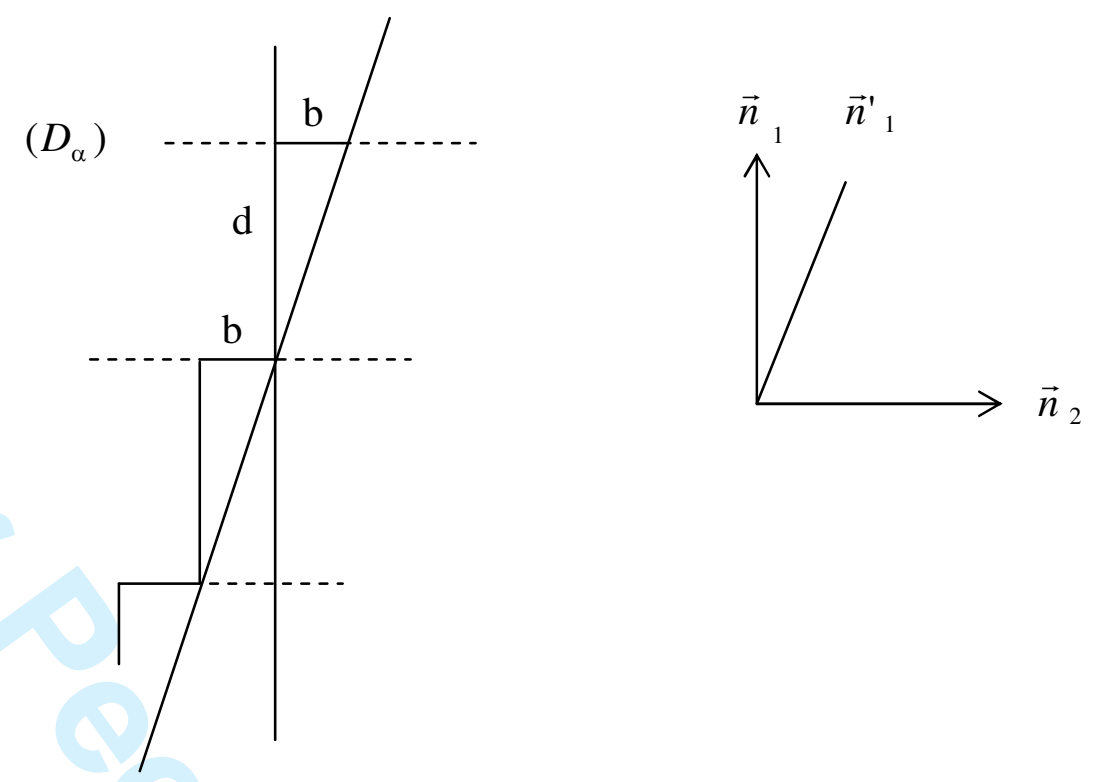

Fig. 3 
Philosophical Magazine \& Philosophical Magazine Letters

Page 4 of 14

1
2
3
4
5
6
7
7
8
9
10
11
12
13
14
15
16
17
17
18
19
20
21
22
23
24
25
2
26
27
28
29
30
31
3

$(\mathrm{D} \alpha)$

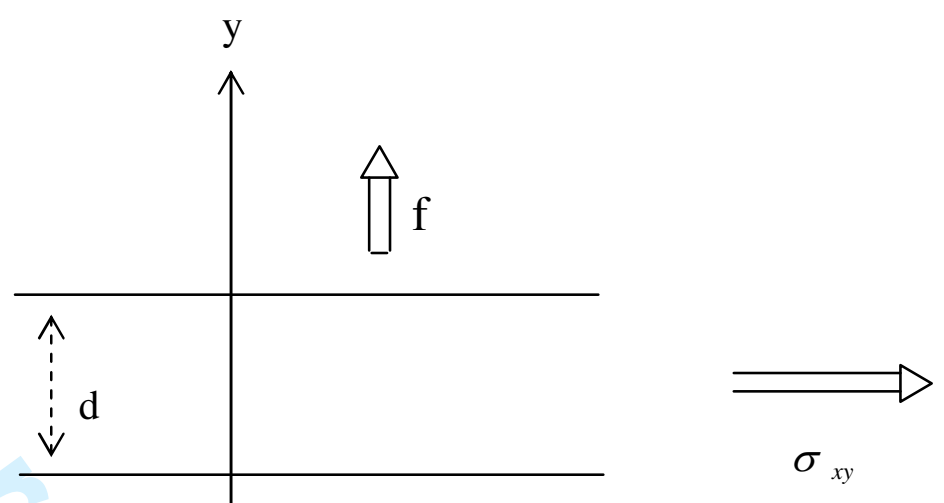

Fig. 4

http://mc.manuscriptcentral.com/pm-pml 
Friction between incommensurate crystals J. Friedel, P.-G. de Gennes

Figure 1

Geometry of the contact plane. The upper layer (director $\vec{n}_{1}$ ) has been rotated by an angle $\theta / 2$, while the lower layer (director $\vec{n}_{1}^{\prime}$ ) has rotated by $-\theta / 2$. Two systems of screw dislocations appear in the contact plane: one of them $D_{\alpha}$ is shown and is parallel to the $x$ axis. The second, $D_{\beta}$, is not shown and is parallel to the $y$ axis; both sets have the same interline distance $d$.

Figure 2

Definition of the directors (unit vectors) inside one layer.

\section{Figure 3}

A ladder of equidistant screw dislocations (with Burgers vector $b$ and distance $d$ ) creates a rotation of the director $\vec{n}_{1} \rightarrow \vec{n}_{1}^{\prime}$.

Figure 4

The stress (along $x$ ) creates a force per unit length $f_{y}$ (along $y$ ) acting on the dislocations $D_{\alpha}$ The dislocations move along $y$, while the atoms move along $x$. 


\title{
Friction between incommensurate crystals
}

\author{
J. Friedel ${ }^{(+)}$, P.-G. de Gennes ${ }^{(*)}$ \\ June 19, 2006 \\ Phil. Mag. MS TPHM 06 Feb 0042 \\ Revised Version
}

\begin{abstract}
We present an overview of friction processes expected between two ideal crystals of strong layers (graphite, $\mathrm{MoS}_{2} \ldots$ ) when one crystal is rotated with respect to the other by a certain angle $\theta$. We assume perfect conditions: no impurities; no preexisting dislocations in the bulk of the crystals; slow gliding velocities. Two regimes show up: a) weak coupling when $\theta>\tilde{U}_{1} / U_{L}$, where $\tilde{U}_{1}\left(U_{L}\right)$ are typical intra (inter) layer interactions. Here we expect weak friction, controled by 2 phonon processes, and analyzed by Sokoloff et al. However we point out that surface waves at the interface also play a role; b) strong coupling $\left(\theta<U_{N} / U_{L}\right)$ where two orthogonal sets of screw disclinations should build up in the contact plane, as shown long ago by F. C. Frank. Here (to a first approximation) the dislocations are arranged in ladders, and we expect solid friction with a Peierls-Nabarro threshold stress.

Keywords - Incommensurate structures - Tribology - Grain boundaries - Rayleigh-Love waves - Graphite

${ }^{(+)}$Bât. 510 Université Paris Sud, 91405 Orsay, France

${ }^{(*)}$ Institut Curie Recherche, Bât. Curie, 26 rue d'Ulm 75231 Paris Cedex 05, France (pgg@curie.fr)
\end{abstract}

\section{Introduction}

Friction Experiments have been performed with an atomic force microscope (AFM) tip sliding on a single crystal surface (eg $\mathrm{NaCl})$ [1]. This system would be relatively simple if the tip was extremely sharp, with a single atom probing the surface - corresponding to the Tomlinson model [2]. However, in practice, the tip is blunt, and the tribological properties are more complex.

Another case is obtained with an AFM tip facing a layered crystal (graphite, $\mathrm{MoS}_{2}$ ). Here, if the tip is rotated by an angle $\theta$, one often finds an unusually small friction [3][4]. This feature is associated with the (misleading) word 'superlubrication'. In these experiments it has been suggested that a flake from the crystal detaches and follows the rotation of the tip [5]. The system may then (in favorable cases) build up an incommensurate structure at the interface between 
flake and bulk. This situation is interesting, but again complex, because (i) the size of the flake may be very small (nanometers): the frictional behavior may depend on the flake size; (ii) the state of the support layer below the contact plane (after the extraction of the flake) is not controled.

Our aim, in the present text, is to discuss theoretically an ideal situation, with two monocrystals of graphite (or $\mathrm{MoS}_{2}$ ) of relatively large contact area (micrometers rather than nanometers). One crystal is rotated with respect to the other by a certain missetting angle $\theta$ (Fig. 1). The situation is assumed to be perfect: no impurities, no dislocations inside each of the crystals.

We start by recalling a crucial distinction between two regimes (Section 2): one decoupled regime where the two crystal arrangements are nearly independent, and one coupled regime where the missetting is compensated by two sets of screw dislocations. In Section 3 we explore the decoupled regime, finding a weak viscous friction as predicted by a number of authors [6][7]. In Section 4 we analyze strong coupling, leading to solid friction. In Section 5 we compare our results with earlier theoretical work.

\section{Strong coupling versus weak coupling}

\subsection{Two ladders of screw dislocations}

The contact displayed on Fig. 1 is a grain boundary lying in the $(x y)$ plane. We know that grain boundaries are realised in practice through ladders of dislocations [8][9]. To construct these ladders in the present case, we start by defining atomic orientations inside one graphitic plane, through two orthogonal unit vectors (or 'directors') shown on Fig. 2. We want to rotate the directors in the top crystal $\left(\vec{n}_{1} \rightarrow \vec{n}_{1}^{\prime}, \vec{n}_{2} \rightarrow \vec{n}_{2}^{\prime}\right)$ by an amount $\theta$.

a) Consider first the shift $\vec{n}_{1} \rightarrow \vec{n}_{1}^{\prime}$. Frank achieves this by a ladder of screw dislocations, each of them creating a step in the atomic lines parallel to $\vec{n}_{1}$ (Fig 3). The magnitude of this step is the Burgers vector $b$ of the dislocation. With a ladder of equidistant dislocations (distance $d$ ). The resulting tilt angle is:

$$
\theta=b / d
$$

b) We introduced one ladder (dislocations parallel to $\vec{n}_{2}$ ) to ensure that $\vec{n}_{1} \rightarrow \vec{n}_{1}^{\prime}$. But we are at this moment obtaining sheared layers, since $\vec{n}_{2}$ is not normal to $\vec{n}_{1}^{\prime}$. To release the shear imposes a second ladder (parallel to $\vec{n}_{1}^{\prime}$ ) with the same spacing $d$ (Eq 1), giving the correct rotation $\vec{n}_{2} \rightarrow \vec{n}_{2}^{\prime}$.

Actually our choice of directions $\left(\vec{n}_{1}\right.$ and $\left.\vec{n}_{2}^{\prime}\right)$ for the two sets of dislocations is clumsy, and unsymetrical. Another choice (equivalent to order $\theta^{2}$ ) and completely symmetrical, is based on the bisector axes $x$ and $y$ of Fig 1 . The two sets of Frank dislocations are parallel to the symmetry axes $(x, y)$ of the rotation pattern. 


\subsection{Dislocations: infinitesimal or quantized?}

If the layers are very rigid, we cannot distort them to build up real dislocations with a well defined core: we must then think of a large number of dislocations ( $d$ small) but each of them being very weak ( $b$ very small). This still allows to comply with Eq 1.

This limit of rigid layers and weak interlayer couplings corresponds to what Frank [8] called infinitesimal dislocations, and to what we call weak coupling. Let us now discuss the opposite limit, where the layers are not infinitely rigid. The contact will then tend to adjust, and to build up dislocations of finite Burgers vector $b$ (where $b$ is now an intralayer repeat distance). Let us estimate what are the energy changes associated with this process, for a ladder parallel to the $x$ axis.

a) One dislocation has an energy per unit length

$$
W \sim \mu b^{2} \ln \frac{d}{b} \sim \mu b^{2}
$$

where $\mu$ is a 3 dimensional elastic modulus, dominated by the intralayer interactions $\mu b^{3} \sim U_{L}$. Note our crude simplifications: ignoring numerical coefficients and dropping the logarithmic factor; also ignoring the difference between intralayer distances: $(b)$ and interlayer distances: $(a)$. Since the interdislocation distance is $d$, the energy per atom in the contact plane associated with Eq 2 is:

$$
b W(b / d) \sim U_{L} b / d
$$

b) On the other hand the dislocations have allowed the atoms in the contact plane to sit just in the right position with respect to their partners in the opposite layer: thus we gained an energy $\sim \tilde{U}_{I}$ per atom, where $\tilde{U}_{I}$ is the interlayer energy per atom. The symbol $\tilde{U}_{I}$ reminds us that $\tilde{U}_{I}$ is not the bare interaction, but a renormalized (smaller) interaction, due to elastic distortions and estimated by Peierls [10]. The quantized dislocation ladder will win if $\tilde{U}_{I}>U_{L} b / d$ or (using Eq 1)

$$
\theta<\frac{\tilde{U}_{I}}{U_{L}}
$$

When the condition 4 is satisfied, we expect to be in the strong coupling regime, with dislocations of finite Burgers vector $b$.

\subsection{Complications due to the incommensurate structure}

If $\theta$ is a rational number, we can accomodate the dislocation ladder as a multiple of the basic period $b$ and the contact is spatially periodic. But we must often deal with non rational values of $\theta$. What happens then?

We shall give here a poor man's approach to this classical problem, and consider, as an example, the following case, with $\theta$ small. Let us define an integer $p$ such that

$$
p<\frac{1}{\theta}<p+1
$$


In a 0 order approximation we can think of the ladders as dimensionless spacing $p$. In a first order approximation we would think of ribbons with $k_{1}$ distances equal to $p$, and $k_{2}$ distances equal to $p+1$; the relative fraction of these two distances being imposed by Eq 1 . To a first approximation we can choose $k_{1}$ and $k_{2}$ as integers (as small as possible) and then generate a periodic structure, of period $\left(k_{1}+k_{2}\right) b$. But this is not exact, since $\theta$ is not rational. We must then go to a second approximation, etc, generating what is often called a devil's stair case.

Physically, the search for an exactground state of piled dislocations is not very meaningful, because the contact will not usually achieve the ideal ground state. The 0 order result $(d=b p)$ is meaningful, but the energy gained by refining the structure is very small (for large $p$ ). Observable arrangements are dependent upon sample history. A practical picture is built with dislocations of distance, $p$ or $p+1$, plus some disorder at this level.

\section{$3 \quad$ Friction in the weak coupling limit}

\subsection{Principles}

When the two incommensurate crystals can be considered as nearly rigid, we expect that, at the crudest level, the interaction energy reaches a constant value by a simple averaging process. But if we go to temperatures $T$ comparable with the Debye temperature $\Theta$ of the crystal, we do expect a viscous friction. The perturbation picture built by Sokoloff [7] for this situation amounts to describe one portion (say: the top part) creating a sinusoidal surface potential

$$
U(\vec{r})=\tilde{U}_{1} \cos \left(\vec{\tau}_{+} \cdot \vec{r}\right)
$$

where $\vec{\tau}_{+}$is a reciprocal lattice vector of the top crystal. (The smallest $\vec{\tau}_{+}$ giving the largest $\tilde{U}_{1}$ ). If we move the top part at velocity $\vec{V}$ in the contact plane, this generates a time periodic potential of frequency

$$
\Omega=\vec{\tau}_{+} \cdot \vec{V}
$$

( $\Omega$ is small because the velocities $V$ of interest are small at the atomic scale). This surface potential will excite some phonons in the bottom part, and thus lead to a viscous dissipation $\frac{1}{2} T S^{\circ}$ (the factor $1 / 2$ accounts for the fact that dissipation occurs in both top and bottom). In many cases this will lead to a dissipation quadratic in velocity

$$
T \stackrel{\circ}{S}=\vec{V} \hat{z} V
$$

where $\hat{z}$ is a $2 \times 2$ matrix, with eigen vectors parallel to the two symmetry axes $(x, y)$ of Fig 1 (In most of our -rough- discussion we shall ignore the anisotropy of $\hat{z}$ ).

The friction stress $\sigma_{z \alpha}$ (where $\alpha$ is $x$ or $y$ ) is then given by

$$
\sigma_{z \alpha}=z_{\alpha \beta} V_{\beta}
$$




\subsection{Phonon processes}

For $T \succ \Theta$ the amplitudes $u$ of the thermal vibration of one atom have an average square

$$
<u^{2}>=b^{2} \frac{k_{B} T}{M c^{2}} \cong b^{2} \frac{k_{B} T}{U_{L}}
$$

where $M$ is the mass/unit, $c$ the sound velocity. $k_{B}$ is the Boltzmann constant. (Eq 9 is a simplified form, ignoring the anisotropy of the crystal). Since $\alpha=$ $(u / b)^{2}$ is small, we can use an expansion in powers of $\alpha$ to discuss the friction. The terms of order $\alpha$ correspond to one phonon processes where the perturbation of Eq 5 creates a single phonon in the bottom crystal, of wave vector $\vec{k}$ and frequency $\omega_{k}$. The requirements of energy conservation and quasi momentum conservation impose $\omega_{k}=\Omega$ and $\vec{k}=\vec{\tau}_{+}-\vec{\tau}_{-}$(where $\vec{\tau}_{-}$is any reciprocal lattice vector of the bottom part). Sokoloff pointed out that these stringent requirements can be satisfied only for a few discrete points in $k$ space: a few phonons can ring, but we do not expect a realistic dissipation.

One must then go to two phonon processes (ie to order $\alpha^{2}$ ). Here a phonon $(\vec{q})$ is absorbed. Another phonon $(\vec{k})$ is excited, and the conservation rules are

$$
\begin{gathered}
\Omega=\omega_{k}-\omega_{q} \\
\vec{\tau}_{+}=\vec{\tau}_{-}+\vec{k}-\vec{q}
\end{gathered}
$$

We have displayed here the (dominant) Raman process where $\omega_{k}$ and $\omega_{q}(\sim$ $\left.10^{12} \mathrm{sec}^{-1}\right)$ are much larger than $\Omega\left(\sim 10^{8} \mathrm{sec}^{-1}\right)$. Here, because we have more degrees of freedom, many events are allowed (with $k$ and $q$ of order $b^{-1}$ ) provided that the whole phonon spectrum is excited (ie $T>\Theta$ ). The (qualitative) answer for the viscous friction stress is then

$$
\sigma=z V \cong(2 \pi)^{3} \theta^{2} \sigma_{0} \frac{k_{e} T}{U_{L}} \frac{V}{c}
$$

where $\sigma_{0}$ is a characteristic stress

$$
\sigma_{0}=\frac{\vec{U}_{1}^{2}}{U_{L}} \frac{1}{b^{3}}
$$

With $\theta=0.1, U_{L}=5 \mathrm{eV}, \vec{U}_{1}=0.5 \mathrm{eV}, T=300^{\circ} \mathrm{K}$, and $V=10^{-2} \mathrm{~cm} / \mathrm{sec}$, we estimate $\sigma_{0}=10^{5} \mathrm{~atm}$ and $\sigma \sim 10^{-4} \mathrm{~atm}$. Thus the viscous friction process is weak: superlubrication is allowed in the weak coupling regime.

We must however emphasize that this conclusion fails if we have a monolayer rather than a bulk crystal, because the singular fluctuations of $2 \mathrm{~d}$ crystals allow for multiphonon processes.[11]

\section{Friction with strong coupling}

\subsection{Geometry of the motions}

Consider now a rotation $\theta$ smaller than $\vec{U}_{1} / U_{L}$. Then we deal with two ladders of screw dislocations, parallel to $x$ and $y$ in Fig 1 . Choose for instance a stress 
$\sigma_{x z}$ on the interface. This will act on the dislocations of Burgers vector parallel to $x$. The force per unit length $f$ on one dislocation will be along $y$ as shown on Fig. 4. If the dislocations can move, with a certain velocity $V_{D}$ along $y$, the contact is slipping, with a velocity $V$ along $x$

$$
V=\frac{b}{d} V_{D}
$$

\subsection{The threshold stress}

At low stresses $\sigma_{x z}$, we do not expect any motion $\left(V_{D}=V=0\right)$ because the dislocation are pinned on the lattice structure by a sinusoidal surface potential (Eq 5). This pinning is reminiscent of the discussion by Peierls on the stress required to move a dislocation along a glide plane [10]. The resulting threshold stress is (ignoring some minor prefactors)

$$
\sigma^{*} \cong \frac{U_{1}}{b^{3}} \exp (-2 \pi a / b)
$$

This displays the renormalisation from the bare sinusoïdal potential $U_{1}$ to the renormalized potential $\vec{U}_{1}$ (due to local elastic adjustments)

$$
\vec{U}_{1}=U_{1} \exp (-2 \pi a / b)
$$

where $a$ is the interlayer spacing (while $b$ is always the intralayer size of one unit). ${ }^{1}$

When $\sigma_{x z}<\sigma^{*}$ we expect no motion of the dislocations and (from Eq 13) no slippage at the contact plane $(V=0)$. Thus strong coupling naturally leads to solid friction. We can actually rederive the criterion for strong coupling (associated with eq. 4) by imposing that the Peierls stress $\tau^{*}$ be larger than the local stress on one dislocation, due to the next one in the ladder.

It is of interest to notice that the system may be highly anisotropic inside the contact plane $(x y)$. For instance, if we impose an oblique stress (where both $\sigma_{z x}$ and $\sigma_{z y}$ are different from 0 ) we may have $V_{x} \neq 0$ if $\sigma_{z x}>\sigma^{*}$ while $V_{y}=0$ if $\sigma_{z y}<\sigma^{*}$. The direction of motion $(\vec{V})$ is not parallel to the direction of the force $\left(\tau_{z \alpha}\right)$.

Ultimately, if both $\sigma_{z x}$ and $\sigma_{z y}$ are larger than $\sigma^{*}$, we can obtain finite velocities $V$ in both directions. Friction is then expected to include both the static stress $\sigma^{*}$ and a dynamic portion $\sigma_{D}$, similar to what is described in Section 3. Usually, we should have $\sigma_{D}<<\sigma^{*}$.

\section{Discussion}

We have defined two regimes: weak and strong coupling, separated by the inequality (4). It is important to notice that the distinction depends not only

\footnotetext{
${ }^{1}$ The dominant Fourier component of the surface forces has a wave vector $2 \pi / b$. There are other components (down to $\sim 2 \pi / d$ ) but the leading term is the first one. This is the basis of equation (16).
} 
of the compared interactions $\left(\vec{U}_{1} / U_{L}\right)$ but also of the missetting angle $\theta$ : small missettings favor strong coupling.

Weak coupling should lead to an anomalously weak viscous friction, described qualitatively by Eqs $(12,13)$. However, we should hasten to mention that another form of viscous friction can show up in strong coupling, if $\theta$ is not exactly equal to the inverse of an integer $p$. Then we expect a certain amount of disorder along the dislocation ladders (with distances being equal to $p b$ or $(p+1) b$. Moving a disordered array is similar to moving impurities in the contact plane. Here there are no selection rules on the wave vectors, and the friction is large -as discussed for instance in Ref [12].

It may also be of interest to mention another feature associated with weak coupling: we expect surface waves to propagate near the interface (within a thickness comparable to the wavelength). These waves are associated with a relative displacement of both lips. The boundary conditions are as follows: a) since the crystals glide on each other, the shear stress $\sigma_{x z}$ at the interface vanishes; b) the normal stress $\sigma_{z z}$ must be continuous: using the mode symmetry, one finds that this imposes $\sigma_{z z}=0$. Thus the boundary conditions coincide with the conditions for Rayleigh-Love waves - describing seismic propagation at the surface of the earth [13]. Love waves should be present within weak coupling. With strong coupling a phonon gap is expected. These features are transpositions of known features for the Frenkel Kontorova model, to be discussed below.

We should now compare our views with a number of theoretical pictures of superlubrication, which have been presented in the past.

\subsection{The Tomlinson model}

This deals with a single atom moving on a crystal surface, and provides some extremely interesting ideas. But it has strong limitations.

(a) It is not easy to go from the single atom picture to the present incommensurate problem -especially in the strong coupling limit, where the distance $d$ between neighboring dislocations plays an important role.

For instance the model calculations of Verhoeven et al [14] do discuss the contact between a flake of finite size (nanometric) and a crystal: they are useful, but restricted to two interacting pieces, each of them being fully rigid: this ignores the possibility of a dislocation network. These calculations should be extended to situations where $d$ is comparable to the flake size.

(b) The Tomlinson model ignores an important feature, pointed out many years ago by Peierls [10]: the crystal structure is deformed by the atom; this deformation leads to a renormalized sinusoïdal interaction of amplitude $\vec{U}_{1}$ much smaller than the bare interaction $U_{1}$. (See Eq 16)

\subsection{Frenkel-Kontorova}

A historic approach on incommensurate systems is the model of Frenkel and Kontorova, for which many exact results are known [15] [16]. Here one speaks of two incommensurate chains (one rigid, one elastic) coupled by a sinusoïdal 
potential due to the rigid part. This can lead to a transition from weak to strong coupling, reminiscent of what we describe here, but there are some important differences.

(a) The Frenkel-Kontorova model is one domensional, and gives a criterium for weak coupling which differs from ours: namely $\theta<\left(\vec{U}_{1} / U_{L}\right)^{1 / 2}$. This is an artefact. In the real case, the elastic distortions are not restricted to two layers, but spread over a number of layers comparable to the interdislocation distance. The correct power law is given by Eq (4). Some studies on a 2 dimensional extension of the Frenkel-Kontorova model have appeared [17].

(b) The discussion of friction is often focused on very high slip velocities $V$ (comparable to the speed of sound). This is of great theoretical interest, but not relevant for classical tribology measurements, where $V \sim 1 \mathrm{~cm} / \mathrm{sec}$ at most.

\subsection{Viscous friction}

The calculations of viscous friction by Sokoloff [7] and others are useful, provided that we do have a weak coupling situation. (With strong coupling, the situation is completely different -dominated by the Peierls Nabarro stress - and the viscous corrections are not very important.) The Sokoloff approach has one weakness however: it does not include the emission of phonons in the form of Rayleigh-Love waves, which may be crucial. We should also remember that: a) if we have impurities on the interface, on some surface roughness, the selection rules (11) drop out and friction is strongly inscreased. This has been discussed recently [18]; b) the Sokoloff conclusions break down if one of the partners is a monolayer (rather than a bulk crystal): then the friction is expected to be large [11].

\section{Conclusions}

On the whole, superlubrication is not a complete surprise, provided that we are in the weak coupling regime $\theta>\vec{U}_{1} / U_{L}$. But the details of the process are rather intricate. We should mention some extra complications.

(i) For hexagonal layers like graphite it is not sure that we keep two orthogonal sets of straight dislocation: the dislocations might tend to adjust to the hexagonal symmetry (at least for small $\theta$ ).

(ii) If the crystals are metallic, we can have another form of viscous friction, due to the excitation of electrons near the Fermi level. Even in graphite, this may play a role, as pointed out to us by a referee.

Both points (i) and (ii) would deserve further investigation.

A final comment: we discussed only the extreme regimes (uncoupled/coupled). The transition between them raises many questions: is it abrupt or continuous? Do we have critical exponents near the transition point? The answers are known for the Frenkel-Kontorova model [16] but (as far as we know), this question remains open for two bulk crystals in contact. 
Aknowledgements - We thank J. M. Martin who introduced us to the problem of superlubrication, C. Girit, who gave us a description of the existing literature, and C. Caroli, for some very useful comments.

\section{References}

[1] A. Socoliuc, R. Bonnewitz, E. Gnecco, E. Meyer, Phys. Rev. Lett. 92, 134301 (2004).

[2] G. A. Tomlinson, Phil. Mag. 7, 905 (1929)

[3] C. Mate, G. McClelland, R. Erlandsson, S. Chiang, Phys. Rev. Lett., 59, $1942(1987)$

[4] M. Dien Wiebel, N. Pradeep, G. Verhoeven, H. Zandbergen, J. Frenken, Surf. Sci. 576, 197 (2005)

[5] S. Morita, S. Fujisawa, Y. Sugawara, Surf. Sci. .. 23, 1 (1996)

[6] J. Sacco, J. Sokoloff, A. Widom, Phys. Rev. B 20, 5071 (1979)

[7] J. Sokoloff, Phys. Rev. B 42, 760 (1990)

[8] F. C. Frank, Pittsburgh Conf. Office of Navel Research 2, 542 (1949)

[9] See for instance J. Friedel, "Dislocations", Pergamon Press (London) 1964

[10] R. E. Peierls, Proc. Phys. Soc. 52, 34-37 (1940)

[11] P.-G. de Gennes, C.R. Acad. Sci. (Paris) Physique 7, 266 (2006)

[12] B. Persson, "Sliding Friction", Springer (Berlin) 2000

[13] See for instance: L. Landau, E. Lifshitz, "Theory of Elasticity", Pergamon Press (London) 1952

[14] G. Verhoeven, M. Dienwiebl, J. Frenken, Phys. Rev. B 70, 165418 (2004)

[15] Y. Frenkel, T. Kontorova, Z. Exp. Th. Phys. 8, 89; 1340; 1349 (1938)

[16] See for instance: M. Peyrard, S. Aubry, J. Phys. C 16, 1593 (1983)

[17] O. Braun, T. Dauxas, M. Palsy, M. Peyrard, Phys. Rev. E 55, 3598 (1997)

[18] U. Tartaglino, V.N. Samoilov, B.N.J Persson, J. Phys.: Condensed Matter 18, 4143 (2006). 